\title{
ABORDAGEM PROPEUDÊUTICAS DE ENFERMAGEM AO PACIENTE COM TRAUMA RAQUIMEDULAR NO PRONTO ATENDIMENTO
}

Vinicius Lino de SOUZA NETO ${ }^{1}$

Márcia Angélica Dantas Jesuíno da $\operatorname{COSTA}^{2}$

Ana Elza oliveira de MENDONÇA ${ }^{3}$

\begin{abstract}
${ }^{1}$ Discente do Curso Bacharelado em Enfermagem da Universidade Federal de Campina Grande - Campus Cuité/Paraíba/Brasil. Membro do grupo de pesquisa em epidemiologia e saúde coletiva- GPESC/UFCG.

E-mail: vinolino@ hotmail.com

${ }^{2}$ Enfermeira pela Universidade Federal de Campina Grande - Campus - Cuité/Paraíba/Brasil.

E-mail: marciadantas@ @ hotmail.com

${ }^{3}$ Enfermeira. Doutoranda em Ciências da Saúde CCS/UFRN, Mestre em Enfermagem pela Universidade Federal do Rio Grande - UFRN. Especialista em Nefrologia pela Universidade Federal de São Paulo - UNIFESP e em Enfermagem em Terapia Intensiva pela FELM/RJ. Professora do curso de Enfermagem do Centro Universitário de Ciências Cultura e Extensão do RN e da Universidade Federal do Rio Grande do Norte - UFRN. E- mail: a.elza@ uol.com.br
\end{abstract}

Contato com autor responsável: Vinicius Lino de Souza Neto

E-mail: vinolino@ hotmail.com

Endereço postal: Rua Santa Clara, № 262, Centro, Campina Grande - PB, Brasil. CEP: 58.400-170.

Recebido em: 30/05/2014 - Aprovado em: 22/09/2014 - Disponibilizado em: 15/12/2014

\begin{abstract}
Resumo
O traumatismo raquimedular é uma agressão à medula espinhal que pode ocasionar danos neurológicos, como alteração das funções motora, sensitiva e autônoma. O presente estudo objetivou retratar a importância das propedêuticas de enfermagem ao paciente com TRM no pronto atendimento e apresenta uma proposta de algoritmo para sequenciar a assistência de enfermagem no pronto atendimento vitimado pela patologia. Trata-se de uma revisão integrativa da literatura, realizada em trê bases de dados da Biblioteca Virtual de Saúde: Literatura Latino Americana e do Caribe em Ciências da Saúde (LILACS) e Base de Dados Bibliográficos Especializada na Área de Enfermagem (BDENF), Medical Literature Analysis and Retrieval Sistem on-line (Medline). Foram selecionados dez artigos, publicados entre 2002 e 2012. A sistematização do atendimento de enfermagem a vitima de TRM no pronto atendimento demanda cuidados específicos, centrados não apenas na sobrevivência do paciente, mas, na prevenção de danos irreversíveis e reabilitação menos sofrida e onerosa tanto no contexto financeiro quanto social.

Descritores: Traumatismos da Medula Espinhal. Traumatismos do Sistema Nervoso. Atendimentos de Enfermagem. Enfermagem.
\end{abstract}

\begin{abstract}
The spinal cord injury is an injury to the spinal cord which can cause neurological damage, such as changes of motor, sensory and autonomous functions. The present study aimed to portray the propaedeutic importance of nursing to patients in the emergency department with SCT and proposes a sequencing algorithm for nursing care in the emergency department victimized by pathology. This is an integrative literature review, conducted in three red databases of the Virtual Health Library: Latin American and Caribbean Literature on Health Sciences (LILACS) and Specialized Bibliographic Data Base in Nursing Area (BDENF), Medical Literature Analysis and Retrieval Sistem Online (Medline). Ten articles published between 2002 and 2012 were selected. Systematization of nursing care to the victim in the emergency room TRM requires specific, focused care not only in the survival of the patient, but to prevent irreversible damage and rehabilitation less painful and costly both the financial and societal contexto.

Descriptors: Spinal Cord Injuries. Injuries to the Nervous System. Nursing visits. Nursing.
\end{abstract}




\section{INTRODUÇÃO}

O numero de pessoas que sofrem de lesão medular aumentou consideravelmente nos últimos anos, estando está situação com o nível de desenvolvimento do país, com isso o processo de urbanização vem modificando o perfil epidemiológico destes países, como o Brasil. Nas ultimas décadas, houve uma crescente queda das doenças infectoparasitárias como causa de morte, ocorrendo um aumento das taxas de morbimortalidade significativa por causas externas, como, homicídio e traumas, originados principalmente pela violência urbana e veículos automotores. ${ }^{1}$

As causas externas como os acidentes de trânsito, queda de altura, ferimentos por arma de fogo, mergulho são as principais causas do TRM documentadas pela literatura, no cenário nacional e mundial. Caracterizado como um agravo emergente o TRM vem aumentando a suas incidências nas ultimas décadas, devido ao crescimento de acidentes indústrias e automobilístico, decorrente do processo de urbanização, responsáveis por lesões complexas e graves da coluna vertebral. $^{2}$

Os jovens são os maiores vitimados por lesões de causa externas. De acordo com a UNESCO, em 1980 as mesmas eram responsáveis por aproximadamente metade do total de mortes dos jovens do país e no ano 2000 acima de 2/3 dos jovens morreram por causas externas. Dentro deste panorama, encontra-se o Trauma raquimedular (TRM), sendo definido como uma agressão traumática a coluna vertebral e os seus componentes. Traumatismos da região torácica, cervical e lombosacra correspondem a 3,3\% como causas de morte por trauma, precedidos pelo traumatismo craniano $10,2 \%$ e lesão de órgãos internos $7,2 \%{ }^{3}$

Nos achados literários é consenso o predomínio do TRM no sexo masculino, podendo ser explicada pelo tipo de atividade desempenhada pelos homens, maior exposição durante o trabalho, no trânsito, em atividades físicas, maior envolvimento em briga e agressões. Em relação à faixa etária, há maior acometimento em adultos jovens, com pico de incidência dos 20 aos 40 anos, na idade produtiva do individuo. ${ }^{4}$

O trauma é o principal fator de causa da lesão medular e ocorre em cerca de $20 \%$ dos casos, com a interrupção do desenvolvimento fisiológico da medula espinhal o individuo pode apresentar déficit motor, sensitivo e autonômico abaixo do nível da lesão, onde o quadro depende do nível da lesão e da sua gravidade, podendo ser parcial ou total. O quadro clinico apresentado pelo paciente com lesão na medula espinhal pode submete- ló a diversas complicações como ulcera por decúbito, pneumonia, infecções do trato urinário, depressão, dentre outras, que dificultam a reabilitação, aumentando o período de hospitalização e consequentemente 
o índice de mortalidade, fazendo com que está lesão seja onerosa e incapacitante. ${ }^{5}$

$\mathrm{O}$ atendimento hospitalar é essencial para o delineamento do quadro clínico do TRM, por isso que a equipe de enfermagem do pronto atendimento das unidades de emergência hospitalar deve está capacitadas, para realizarem uma abordagem assistencial lógica e precisa ao individuo, e assim aumentando a sua expectativa de vida diante da patologia. A propedêutica em enfermagem se traduz por ações desenvolvidas pelo enfermeiro, a saber, história clínica, diagnóstico de enfermagem, prescrição da assistência, evolução e registro de enfermagem, todas essas etapas são desenvolvidas de forma sistematizada. Assim, compreende-se por propedêutica ou processo de enfermagem, a utilização de uma abordagem organizada para alcançar um propósito. Diante do exposto, verifica-se a necessidade de descrever a importância da abordagem propedêutica de enfermagem frente ao paciente com TRM sobre a visão de um algoritmo de conduta assistencial no pronto atendimento. ${ }^{6}$

Diante de tudo exposto, o estudo em tela teve como objetivo de retratar a importância das propedêuticas de enfermagem ao paciente com TRM no pronto atendimento.

\section{METODOLOGIA}

Trata-se de um estudo de revisão integrativa da literatura, organizada a partir das seguintes etapas: estabelecimento da hipótese e objetivos da revisão; estabelecimento de critérios de inclusão dos artigos; definição das informações a serem extraídas dos artigos selecionados; seleção dos artigos; análise dos resultados; discussão dos achados e apresentação da revisão. ${ }^{7}$

A hipótese surgiu através do questionamento dos pesquisadores frente à falta de conhecimentos científicos que retrate o foco da pesquisa, levando ao direcionamento da questão, que foi: Qual a relevância da propedêutica e o algoritimo de conduta assistencial de enfermagem frente ao individuo vitimado por TRM no pronto atendimento?

A identificação do objeto de estudo na literatura foi realizada inicialmente a busca nas seguintes bases de dados indexadas a Biblioteca Virtual em Saúde (BVS): Literatura Latino-Americana e do Caribe em Ciências da Saúde (LILACS), Medical LiteratureAnalysisandRetrievalSistem on-line (Medline), Bancos de Dados em Enfermagem (BDENF).

Foram utilizados em um primeiro momento da pesquisa, para a busca dos artigos, os seguintes descritores: "Atendimentos de Enfermagem"; "Traumatismos da Medula Espinhal"; "Traumatismos do Sistema Nervoso", 
conforme explana a Tabela 1. Após a seleção, os artigos foram lidos na integra e selecionados de acordo com os seguintes critérios de inclusão: artigos publicados em português ou resumo de artigos que retratassem o tema em questão; publicados e indexados nas bases e banco de dados pesquisados nos últimos dez anos. amostra final, conforme disposto na Tabela 2, a seguir:

Tabela 2 - Distribuição dos artigos selecionados nas bases de dados MEDLINE, LILACS, BDENF, segundo critérios de inclusão, 2012.

\begin{tabular}{ccccccccc} 
CRITÉR & MEDLI & LILACS & BDENF & Total \\
IOS & \multicolumn{2}{c}{ NE } & & & & & & \\
& $\mathrm{n}$ & $\%$ & $\mathrm{n}$ & $\%$ & $\mathrm{n}$ & $\%$ & $\mathrm{~N}$ & $\%$ \\
Artigos & 04 & 3,2 & 3 & 26, & 3 & 24, & 6 & 54, \\
das MEDLINE, & 5 & 3 & 82 & 0 & 44 & 7 & 51 \\
ultimos & & & & & & & &
\end{tabular}

Tabela 1 - Distribuição dos artigos científicos localizados nas baş@S MED
LILACS, BDENF, segundo os descritores isolados, $2012 . \quad$ ultimos

\begin{tabular}{|c|c|c|c|c|c|c|c|c|c|c|c|c|c|c|c|}
\hline \multirow[t]{2}{*}{ DESCRITORES } & \multicolumn{2}{|c|}{ MEDLINE } & \multicolumn{2}{|c|}{ LILACS } & \multicolumn{2}{|c|}{ BDENF } & \multicolumn{2}{|c|}{10 angsotal } & \multirow{3}{*}{$\begin{array}{c}2,4 \\
3\end{array}$} & \multirow{3}{*}{$\begin{array}{l}2 \\
5\end{array}$} & \multirow{2}{*}{$\begin{array}{l}20 \\
32\end{array}$} & & \multirow{2}{*}{$\begin{array}{l}14 \\
63\end{array}$} & \multirow{2}{*}{$\begin{array}{l}4 \\
6\end{array}$} & \multirow{2}{*}{$\begin{array}{l}37 \\
38\end{array}$} \\
\hline & $\mathrm{n}$ & $\%$ & $\mathrm{n}$ & $\%$ & $\mathrm{n}$ & $\%$ & $\underset{N}{\text { Titulo }}$ & $\begin{array}{l}03 \\
\%\end{array}$ & & & & & & & \\
\hline Atendimentos de & 00 & 00 & 72 & 0,26 & 50 & 0,18 & $\begin{array}{l}\text { (Portugu } \\
122 \\
\text { ês) }\end{array}$ & 0,44 & & & 32 & & & 6 & 38 \\
\hline Enfermagem & & & & & & & Resumo & 03 & 2,4 & 0 & 2,4 & 0 & 3,2 & 1 & 8,1 \\
\hline Traumatismos da & 25.947 & 96,96 & 394 & 1,47 & 50 & 0,18 & 26.391 & 98,61 & 3 & 3 & 3 & 4 & 5 & 0 & 1 \\
\hline $\begin{array}{l}\text { Medula Espinhal } \\
\text { Traumatismos do }\end{array}$ & 128 & 0,47 & 128 & 0,47 & 5 & 0,01 & $\begin{array}{l}\text { Seleção } \\
261 \\
\text { Final }\end{array}$ & $\begin{array}{c}03 \\
0,95\end{array}$ & $\begin{array}{c}8,1 \\
1\end{array}$ & $\begin{array}{l}0 \\
3\end{array}$ & $\begin{array}{l}49 \\
57\end{array}$ & 4 & $\begin{array}{l}42, \\
32\end{array}$ & $\begin{array}{l}1 \\
0\end{array}$ & 100 \\
\hline
\end{tabular}

TOTAL

$\begin{array}{lllll}26.075 & 97,43 & 594 & 2,2 & 105\end{array}$

0,37 FONTE: dados da pesquisa, 2012

Fonte: dados da pesquisa, 2012.

As estratégias utilizadas para o levantamento dos artigos foram adaptadas para cada uma das bases de dados, de acordo com suas especificidades de acesso, tendo como eixo norteador a pergunta e os critérios de inclusão. Durante a seleção, alguns artigos foram excluídos após a leitura dos resumos, por não se enquadrarem nos critérios de inclusão propostos, outros foram selecionados e excluídos após a leitura do artigo na íntegra, também por não estarem de acordo com os critérios previamente estabelecidos. As buscas foram realizadas entre 10/05/2012 a 10/06/2012, de 26.774 estudos disponibilizados, 10 artigos constituíram a
Posteriormente à seleção dos artigos foi feita a categorização dos estudos para que os dados empíricos concernentes ao objeto e resultados dos estudos pudessem ser descritos e analisados, sendo esta a terceira etapa. Em seguida, na quarta etapa, deu-se início ao procedimento de análise das informações com base no instrumento elaborado, onde a análise dos estudos selecionados, em relação ao delineamento de pesquisa, pautou-se em Polit, Beck, Hungler (2004) e Lo Biondo-Wood, Haber (2001), sendo substituídos os itens do original em inglês citation e interventions por ano de publicação, tipo de estudo e caminho metodologico, na qual tanto a análise quanto a síntese dos dados extraídos dos artigos foram realizadas de forma descritiva, possibilitando 
observar, contar, descrever e classificar os dados, com o intuito de reunir o conhecimento produzido sobre o tema explorado na revisão. ${ }^{8}$

\section{RESULTADOS E DISCUSSÃO}

O Relato mais antigo sobre um paciente com Traumatismo Raquimedular (TRM) foi no ano de 3000 a.C encontrado no Papiro de Edward Smith, presente na grande pirâmide de Gizeh, o documento se refere ao caso de um individuo que sofrera $o$ deslocamento das vértebras do pescoço, e não tinha movimento das pernas e dos braços, não conseguia esvaziar a bexiga e não deveria ser tratado, designado como uma patologia sobrenatural conforme relatos da época ${ }^{9-11}$

A medula espinhal consiste no segmento mais caudal do Sistema Nervoso Central, distribuindo-se da base do crânio até uma região afunilada da medula, denominada cone medular, no nível da primeira vértebra lombar. Ela se divide em tratos longitudinais de axônios, constituindo a substância branca, que circundam regiões centrais de substância cinzenta, na qual está localizada a maior parte dos corpos celulares dos neurônios espinhais. Trata-se de uma estrutura de transmissão de mensagens motoras e sensitivas, entre $\mathrm{o}$ encéfalo. A coluna vertebral é formada por trinta e três (33) a trinta e quatro (34) vértebras (07 cervicais, 12 torácicas, 05 lombares, 05 sacrais e 04 ou 05 coccígeas) A medula espinhal, nos adultos, possui cerca de
$45 \mathrm{~cm}$ e estende-se desde a altura do atlas (C1) até a primeira ou segunda vértebra lombar. $^{12}$

A localização anatômica da lesão está diretamente relacionada ao mecanismo de trauma e cerca de 2/3 das lesões medulares estão localizadas no segmento cervical. As lesões da medula na região torácica ocorrem em $10 \%$ das fraturas desse segmento e em $4 \%$ das fraturas da coluna toracolombar. Elas podem ser completas ou incompletas; caracterizando os quadros de tetraplegia (cervicais C4; C5 e C6); paraplegia (Torácicas e Lombares) ou compatibilidade com a vida quando acometem de $\mathrm{C} 1$ a $\mathrm{C} 3$. As lesões incompletas são classificadas conforme a região medular atingida e a manifestação clínica dependerá do local da mesma. ${ }^{13}$

Os sinais e sintomas do TRM dependem do nível da lesão; o comprometimento neurológico, paralisias ou apenas diminuição de força muscular (paresia), ou as alterações sensitivas como anestesia, diminuição da sensibilidade e parestesias (formigamento, amortecimento, etc), ocorrem geralmente abaixo do nível da lesão. $\mathrm{O}$ individuo também pode apresentar como sintoma a dificuldade de respirar e andar, ressaltando que as primeiras 48horas pos-trauma é fundamental para a sobrevida do paciente. ${ }^{14} \mathrm{O}$ atendimento precoce e correto diminui as sequelas dos traumas medulares, por isso que a equipe do atendimento pré, intra e pós-hospitalar devem ser capacitada 
para o atendimento, principalmente a equipe de enfermagem. ${ }^{15}$

As propedêuticas assistências de enfermagem que engloba o saber clínico, diagnóstico, prescrições de enfermagem, quando aplicadas de forma sistematizadas torna-se substanciais, nas primeiras 48 horas, para a sobrevida do individuo vitima do trauma raquimedular, pois uma equipe bem qualificada permeia uma assistência fluida, com praticas segura, rítmicas e eficazes. ${ }^{16}$

Nesta perspectiva, torna-se fundamental ampliar os cuidados ao paciente vítima de TRM. De modo geral, os indivíduos vítimas de traumatismo raquimedular (TRM) requerem longo período de hospitalização, com tratamento e reabilitação por tempo indeterminado, necessitando que os profissionais envolvidos em seu cuidado tenham a capacitação necessária para atuar na superação da fase aguda do TRM, de modo a facilitar ao cliente, nas fases subsequentes, o alcance de melhor qualidade de vida. ${ }^{17-19}$

Com tudo isso exposto, o presente estudo tende a levar um aprofundamento tecnocientifico aos profissionais que prestam assistência a pacientes vítimas de Trauma Raquimedula, desde o pré, intra e póshospitalar, procurando estabelecer a importância da cascata propedêutica assistencial de enfermagem a esses indivíduos, onde o planejamento das ações através do uso do raciocínio clínico, do processo de enfermagem a pacientes críticos possibilita um melhor direcionamento da assistência, otimizando tempo e garantindo um processo inovador e de qualidade. ${ }^{20}$

\section{CONSIDERAÇÕES FINAIS}

$\mathrm{O}$ trauma raquimedular está associado a traumas de grande energia, como quedas de altura e ou acidentes automobilísticos, com altos índices de sequelas e óbitos. O material disponível na literatura, reforça que as vítimas de trauma devem ser socorridas e transportadas com segurança aos serviços de referência, e atendidas prontamente, pois, toda vítima de trauma é um lesado medular em potencial e não pode ser negligenciados na sala de emergência. Assim, o atendimento pré, intra e pós-hospitalar a esses indivíduos, realizado por profissionais de enfermagem deve ter um teor sumarizado e completo, sem falhas ou incoerências.

A atuação do enfermeiro com capacitação e treinamento específicos é de fundamental importância para o atendimento às vítimas com TRM, por isso, que uma abordagem propedêutica assistencial de caráter lógico ao individuo no pronto atendimento, reflete em uma diminuição das causas secundária do trauma e o aumento da sua sobrevida social, onde todas essas ações podem ser compactadas em um algoritmo de conduta, com isso proporciona um melhor entendimento da equipe no processo assistencial sistematizado. 
Apesar da relevância da temática para enfermagem, observou-se, que ainda é um assunto pouco estudado de acordo com o número de artigos produzidos e publicados por esses profissionais. No entanto, as abordagens temáticas estudadas sobre TRM trouxeram discussões que enveredaram para a contemplação da prática de enfermagem pautada no individuo, com enfoque nos âmbitos assistenciais e de ensino.

\section{REFERÊNCIAS}

1. Amato MCM, Santos MV, Weltson A, Oliveira RS, Boullosa JLR;

Traumatismos Raquimedulares

penetrantes em adolescentes;

COLUNA/COLUMNA. [periodico na internet].2009 Jun/Ago [citado 2012 Maio 10];8(3):323-329. Disponível em:

http://200.144.190.38/bitstream/handle /2012.1/8205/art_AMATO_Traumatis mos_raquimedulares_penetrantes_em _adolescentes_2009.pdf?sequence $=1$

2. Bruni, D.S, Strazzieri, K. C, Gumieiro, M. N, Giovanazzi, R, Sá V. G, Faro, A. C. M. Aspectos

fisiopatológicos e assistenciais de enfermagem na reabilitação da pessoa com lesão medular; Rev. esc. enferm. [períodico na internet] 2004 Mar/Jun [citado 2012 Maio 12]; USP, São Paulo, v. 38, n. 1, mar. 2004.Disponível
Embora os resultados desse estudo tenham sido satisfatórios, torna-se uma necessidade premente o desenvolvimento de novas pesquisas por enfermeiros, que contextualizem enfoques assistenciais específicos da enfermagem frente ao individuo acometido pelo TRM, com ênfase na promoção, prevenção e recuperação da saúde e que venham subsidiar e respaldar as ações profissionais.

em:http://dx.doi.org/10.1590/S008062342004000100009 .

3. Souza LC, Ortega MR, Junior Ribeiro MAF. Trauma Raquimedular e Choque Neurogênico: manifestações incomuns de instabilidade hemodinâmica - como diagnosticar e tratar corretamente;emergência clínica[períodico na internet] 2010; [citado 2012 Maio 14]; 05 (24): 87-89. Disponível em: http://www.sumarios.org/sites/default/ files/pdfs/48626_5795.PDF

4. Waiselfisz J. Mapa da violência III. Organização das nações Unidas para a Educação, a Ciência e a Cultura UNESCO. Brasil.[períodco na internet].2002. [citado 2012 Maio 14]. Disponível em: http://www.agende.org.br/docs/File/da dos_pesquisas/violencia/Mapa\%20da \%20violencia\%20$\% 20 f e v \% 202002$.pdf 
5. Campos, Marcelo Ferraz de et al; Epidemiologia do Traumatismo da Coluna Vertebral; Rev. Col. Bras.

Cir.[ períodico na internet].2008

Mar./Abr. [citado 2012 Maio 12]; Vol.

35 - No 2. Disponivel em:

http://www.scielo.br/scielo.php?script

=sci_arttext\&pid=S0100-

69912008000200005

6. Rede SARAH de Hospitais do Aparelho Locomotor. Lesão medular. [períodico na internet] 2008. [citado 2012 Junho 10]. Disponível em http://www.sarah.br/paginas/doencas/ po/p_08_lesao_medular.htm

7. Del Bel EA, Silva CA, Miladnic M; O trauma raquimedular

COLUNA/COLUMNA. [períodico na internet]. 2009 Nov; [citado 2012 Maio 12]; 8(4): 441-449. Disponível em:

http://200.144.190.38/bitstream/handle /2012.1/11131/art_DEL_BEL_O_trau ma_raquimedular_2009.pdf?sequence $=1$

8. Mendes KDS, Silveira RCCP, Galvão, CM. Revisão Integrativa: método de pesquisa para a incorporação de evidencias na saúde e na enfermagem. Texto Contexto Enferm. [períodico na internet]. 2008; [citado 2012 Maio 12]; 14(4): 758-64. Disponível em: http://www.scielo.br/pdf/tce/v17n4/18. pdf

9. Polit DF, Beck CT, Hungler BP. Fundamentos de pesquisa em enfermagem: métodos, avaliação e utilização. 5a ed. Porto Alegre (RS): Artmed, 2004.

10. Lo Biondo-Wood G, Haber J. Pesquisa em enfermagem: métodos, avaliação crítica e utilização. 4a ed. Rio de Janeiro: Guanabara Koogan; 2001.

11. Amaral, Maria Teresa Mendonça Pinto; Encontrar um novo sentido da vida: um estudo explicativo da adaptação após lesão medular. Rev.Esc.Enferm [períodico na internet]2009 Fev/ Nov; [citado 2012 Maio 12]. 43(3): 573-80. Disponível em:

http://www.scielo.br/pdf/reeusp/v43n3 /a11v43n3.pdf

12. Guyton, A.C.Tratado de Fisiologia Médica. 6a edição. Editora Guanabara, Rio de Janeiro, 2006.

13. Smeltzer, Suzanne C.; BARE Brenda G. Tratado de Enfermagem MédicoCirúrugica. 8. ed. Vol 01 e 04. Rio de Janeiro: Editora Guanabara Koogan, 2002.

14. Siscão, Marino; Marita P.; Cristiano P.; Roberto L.C. A.; Marcos H. D.A. F; Laís H.C.; Trauma Raquimedular: Caracterização em um Hospital 
Público; Arq Ciênc Saúde [períodico na internet] 2007 Jul-Set; [citado 2012 Maio 12] 14(3): 145-7. Disponível em:

http://www.cienciasdasaude.famerp.br /racs_ol/vol-14-3/IIIDDD195.pdf

15. Rodrigues EB, Duarte R, Maia R, Ribeiro J, Vaz H, Pereira E;

Corticoterapia em altas doses no traumatizdo medular - benefício ou prejuízo; COLUNA/COLUMNA [períodico na internet]. 2010 Out; [citado 2012 Maio 12] 9(4): 462-465. Disponível em: http://www.plataformainterativa2.com /coluna/html/revistacoluna/volume9/C oluna_v9n4_462-465.pdf

16. Cafer CR, Barros ALBL, Lucena AF, Mahl MLS, Michel JLM. Diagnósticos de enfermagem e proposta de intervenções para pacientes com lesão medular. Acta paul. enferm. [períodico na internet] 2005 Fev/Abr; [citado 2012 Maio 12] São Paulo, v. 18, n. 4, dez. Disponível em http://www.scielo.br/scielo.php?script =sci_arttext\&pid=S0103-
$21002005000400002 \& \operatorname{lng}=$ pt\&nrm=is o

17. Brito, Luciane Maria Oliveira et al . Avaliação epidemiológica dos pacientes vítimas de traumatismo raquimedular. Rev. Col. Bras. Cir. [períodico na internet] $2011 \mathrm{Set} / \mathrm{Nov}$; [citado 2012 Maio 12] v. 38, n. 5, out. 2011. Disponível em http://www.scielo.br/scielo.php?script $=$ sci_arttext\&pid=S0100$69912011000500004 \& \operatorname{lng}=$ pt\&nrm $=$ is o

18. ATLS/SAVT. Manual do Curso para Alunos. Suporte Avançado de Vida no Trauma. Colégio Americano de Cirurgiões Comitê de Trauma. $9^{\circ}$ ed. Chicago. EUA, 2009. Traduzido e impresso com autorização do American College of Surgions, Brasil, 2009.

19. Elisa, M.S. H; Álvaro, N.A. Guia de Medicina de Urgência. Ed.Manole. ed.2;Baureri-SP,2008

20. Nasi, L.A. Rotinas em ProntoSocorro; Ed.Artmed, ed.2; Porto Alegre, 2005. 